\title{
Research and Design on Real-time Tracking and Controlling Data Processing System Based on Multi-Agent
}

\author{
Libing Guo, Xinbing Fang, Xiangming Li, Wen Mao \& Wei Huang \\ China Satellite Maritime Tracking and Controlling Department, China
}

\begin{abstract}
This paper analyzes the technical features of agent and the main business of aerospace tracking ship tracking and controlling data processing system; establish a multi-agent system model of real-time data processing; take example of USB data processing of trajectory measurement, describes the running and interactive process of the multi-agent subsystems, put forward several principles of how to ensure the consistency of data processing system; through multiple real-time processing validation of satellite task data show that the system can efficiently and accurately identify abnormal of control equipment and flying targets, and has better active, adaptive and alternant.
\end{abstract}

KEYWORD: Agent; Real-time Processing; Model; Monitoring Data

\section{INTRODUCTION}

Aerospace tracking ship tracking and controlling data processing is large-scale, complex system, and has many uncertainties in the process of data processing (Xu Xiaolong et al, 2013). Thus, tasks the complex data processing into several sub-tasks, each subsystem can use their knowledge and problemsolving methods (Zhou Yipeng, 2013), or work together to solve complex data processing problems respectively, can effectively improve efficiency and stability of the system.

Agent has the independence, learning and alternant, be able to take the initiative to complete a task under certain circumstances. Multi-agent systems (MAS) (Huang Min fine, 2013) (Paul C et al, 2003) is composed of a collection of multiple agent, multiagent technology developed with intelligent solving for some large system (Pallis, G et al, 2006), this technology takes advantage of the characteristics of the agent, constitute dispersed and relatively simple problem-specific subsystems through the description, decomposition and distribution of the problem domain, and each subsystem solved the problem collaboratively, this idea is very suitable for the field of aerospace tracking and controlling data processing system, provides a potential way to design and implementation of monitoring data processing of the large-scale aerospace system (Dejun Wang et al, 2007).

\section{CHARACTERISTIC OF AGENT}

Typically, agent contains the following general characteristics: independence, inductive, adaptability, interactivity, collaborative, intelligence and communications capabilities. Agent contains the characteristics is different for the different areas of application. This paper uses of the independence, induction, adaptability, collaboration, intelligence and communications capability characteristics of agent.

A single agent is mainly composed of three parts: data processing, machine learning and knowledge repository of. As shown in Figure1.

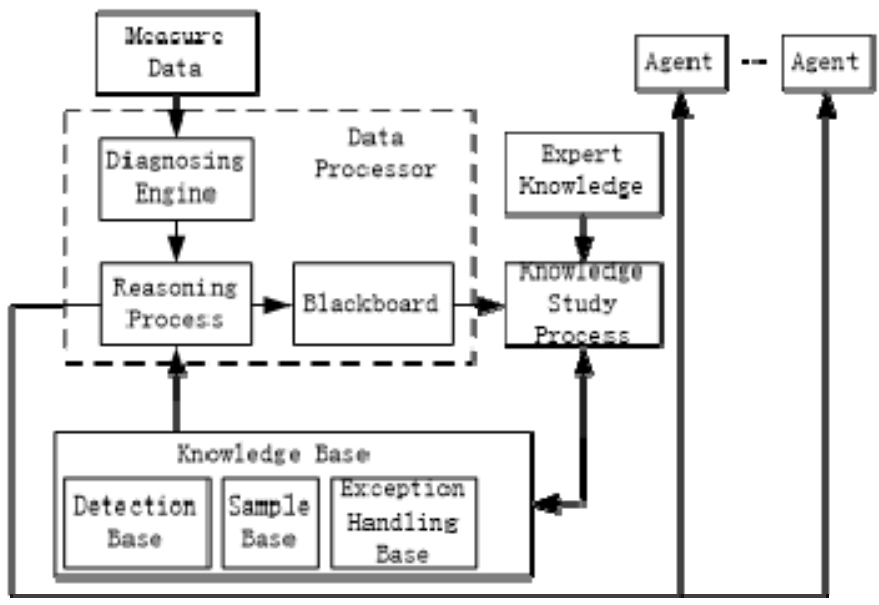

Figure1 A Single Agent Model 
Interactive of process environment: data processor receives the information of the object to be processed and sent the parameters to the processing engine; processing engine diagnosis and treatment based on the knowledge of the knowledge base, finds out whether the measurement data is abnormal, what exceptions and the decision of exception handling, realizes a variety of specific data analysis and algorithms, the processing result is output to the lower sub-task agent, sending the execution state to the system control agent, and the processing result is transmitted by the board to the knowledge of machine learning.

Knowledge learning engine: Knowledge learning engine embodies the interaction of the agent and learning environment. Learning process consists of two parts: one is build the knowledge base based on the existing expertise knowledge, and the second is the perception of new information from the learning environment in the continuous data processing, modified and expanded the knowledge base according to the actual situation, so you can extend the knowledge and improve the capabilities of the agent processing.

Processing Knowledge: Knowledge base is composed of detection base, unusual sample base, exception handling base. Detection base used to identify whether the measurement data is abnormal, detects the measure data, comparing and classifying the error information detected, including the test parameters, the detect model selection, the detection result descriptions; the abnormal sample base used to identify the type of exception, including the choice of abnormality diagnosis algorithm, diagnostic reasoning process, diagnostic results interpret, etc.; the exception handling base used to control and process the exceptions, including the seriousness of the discrimination exception, the eliminate exception processing algorithms and the alarm mode.

\section{AEROSPACE TRACKING SHIP DESIGN FEATURES AND REAL-TIME MONITORING AND CONTROL OF DATA PROCESSING SYSTEM}

\subsection{Operation Analysis}

Aerospace tracking ship equips a variety of monitoring and control equipment containing S-band, Cband, $\mathrm{X}$-band microwave unified monitoring system (USB, UCB, UXB), pulse radar, GNSS, inertial navigation system. The basic process of data processing is as follows:

Receive original data: collect data of each measurement and control devices according the data and time periods driven method respectively. Because of different equipment and different flying targets, their collecting frequency are different the frequency is 50ms, 256ms, 512ms, 2 second, 4 second, and others.

Check the quality of the data: checks the quality of the collected measurement data.

(1) Fitting or alarm processing according to the single abnormal data;

(2) Alarm processing according to the multiple abnormal data directly;

(3) Fitted up points or alarm processing for a small amount of lost points data or dropped frames based on different data;

(4) Alarm processing according to the continuous lost points data directly.

Data matching fusion: carried out time matching process after data health check, to ensure that the different elements of the same kind data at the same time coordinate, given alarm information of the abnormal data anomaly.

Data processing: different types of health of the situation based on the data using different mathematical models for processing, released different alarm information depending on the circumstances of the abnormal data, given recommendations for some fault handling.

Communication process: the data processing results will be sent to the tasks command center related, after receiving and processing the command center directing information, it will be sent to the relevant monitoring and control equipment, monitored and controlled equipment and flight control.

The results show and the local command and control: display various types of data processing results and mission command center information, according to the results released locally directing strategy for the disposal of alarm information and take appropriate measures.

\subsection{System Model}

From the above analysis, the process of the monitoring data real-time processing system of space tracking ship is complicated ,and the system is huge, but the agent is independence, induction, adaptability, collaboration and intelligence, it can be good to finish all types of measurement data processing, and provide data status information, learn data processing knowledge, decompose the complex large system using the MAS, complete various types of measurement data process using the results of data processing and data status information of each agent, and disposal system abnormality appropriately.

So you can build MAS model of the space tracking ship monitoring real-time data processing system, as shown in Figure2: 


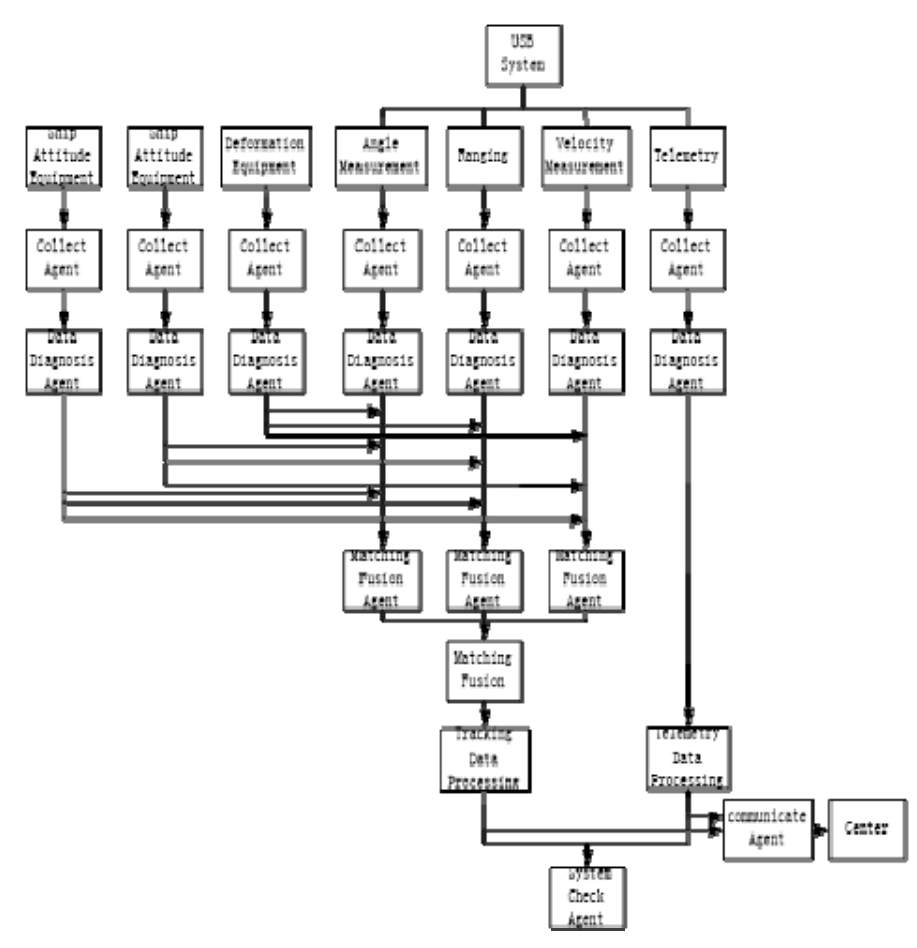

Figure 2 Space Tracking Ship Monitoring Real-time Data Processing System

Each agent's functions are described below:

Data collection agent: according to the IP address and the network devices, receive the measurement data access to the data acquisition frequency and data acquisition mode of each control device, and store it in the respective data buffer for the data diagnosis agent call handling.

Data diagnostic processing agent: diagnose the health degree of collected measurement data in accordance with the rules of each monitoring device data, deal with the abnormal data in accordance with the appropriate disposal rules, the general process is as follows:

(1) Check the data structure: compare the structure of the collected measurement data with the interface protocols specified, if it is accordant then go to the next step; if not, then record the data status information, judged it is invalid measurement data of the monitoring and control equipment, alarm information and notification system control agent for disposal.

(2) Timing checks: Timing checks carry through according to the collected time, if the time interval of the data is greater than the natural spacing can be judged to have dropped in the middle, the outer interpolation method can be used to makeup frame and cancel the validity of the current frame, if the interval is less than the natural, it is judged that the measurement data is reduplicate frame, and the inner interpolation method can be used to makeup frame and cancel the validity of the current frame, if the current time is less than the previous data frame time, it is judged the data is out of order, when out of order or disorder appear to judge the monitoring and control of equipment is fault, then send alarm message and notification system control agent for disposal.

(3) Data value check, check the data elements of each measurement frame based on the respective value range, if it does not meet the range of values, and then filter and fit the data according to the mathematical model and records the status information.

Data matching fusion agent: Matching the time of plurality data elements, carried through data interpolation or curve fitting of data for different sampling frequency measurement data, to ensure the data elements involved in the processing are in the unified time coordinate system.

Data processing agent: correct the monitoring and control equipment system error of the measurement data, boat pose, position, distortion correction, calculating the trajectory data (time, location, speed) and instantaneous station site data (time, distance, angle, speed) of the flight target using the corresponding mathematical model, converted the coordinate of the measured data to the horizon line, in order to be consistent with the target coordinate of the entire monitoring system;

Communication agent: assembling the processing result to the data frame according to the data exchange interface, transmitted it to the corresponding task command center at the specified address, and unpack the task command center data received, sent to the corresponding processing module in accordance with the purposes of various types data.

System control agent: It is responsible for monitoring, distributing, scheduling, controlling, and dealing with each agent, to monitor the implementation of the task, receive alarm information for each sub-task agent, and send the results back to the related subtasks agent, each sub-task agent control implement appropriate treatment according to the control information.

\section{CONCLUSION}

The advantage of using multi-agent technology for aerospace tracking and controlling real-time data processing system is very clear.

(1) Building multi-agent systems technology not only improves the stability of the system, and each sub-task agent is dynamical execute, the task has a certain relevance and independence, improves the efficiency of the system, it is general applicable of aerospace control systems.

(2) The intelligence of the system is not only reflected in the single sub-task agent, also reflected in the interaction and collaboration mechanisms of multi-agent.

(3) In the process of the system operation, the expertise knowledge and environment knowledge will be integrated into the system continuously, enrich the 
system knowledge base and improved intelligent systems constantly.

Through the real-time data processing and validating of the satellite launch and control tasks, it shows that the system can efficiently and accurately identify abnormal control equipment and flying targets, with better adaptability, initiative, and interactive, it has good application prospects in the field of aerospace monitoring.

\section{REFERENCES}

Dejun Wang, Linpeng Huang, JianKun Wu, Xiaohui Xu. A Hierarchy Model for Dynamic Replacing of Distributed Components. French Polynesia, ADVCOMP 2007:49-54.

Huang Min fine, GUO Chao-zhen. Failure Multi-Agent-based fault-tolerant middleware. IT'S APPLICATIONS, 2013, 17:72-75.

Pallis, G, Vakali, A.Insight and Perspectives for Content Delivery Networks. Communications of the ACM.2006, 49(1):101-106.

Paul C, Felix B, Len B, et al. Documenting Sofware Architectures. Beijing: TSINGHUA University Press, 2003.

$\mathrm{Xu}$ Xiaolong, Tang Yu, Yang Geng a Mobile Agent Based Distributed Multi malicious processes cooperate to identify the mechanism [J]. Application Research of Computers, 2013, 10 (30):3102-3105.

Zhou Yipeng. Image recognition based on multi-Agent evolutionary computing [J]. Complex Systems and Complexity Science, 2013, 10 (3):55-59. Research Processing System [J] 